\title{
Scheduling Packets for Event-Triggered Control
}

\author{
Maben Rabi, Karl H. Johansson \\ School of Electrical Engineering \\ Royal Institute of Technology (KTH) \\ 10044 Stockholm, Sweden \\ firstname.lastname@ee.kth.se
}

\begin{abstract}
For a single control loop with communication rate constraints, Event-triggered control often outperforms periodic control. When multiple loops are being controlled over a shared contention-based medium, however, the advantage of event-triggered policies is less well understood. In this paper, we consider event-triggered impulse control under lossy communication. The sampling events are determined by level crossings of the plant output. It is shown how a stochastic control criterion depends on the level thresholds and the packet loss probability for a class of integrator plants. For multiple control loops, this result is used to derive a design guideline on how to assign the levels that lead to optimal use of the available communication resources. It is shown that the structure of the event generator depends critically on the loss probability.
\end{abstract}

\section{INTRODUCTION}

There is a tight interaction between the control architecture and the communication protocols in networked control systems. Periodic time-triggered control loops are natural to use together with a TDMA scheduled communication medium, while aperiodic event-triggered control loops are suitable for contention-based medium access [1]. Only recently has a thorough study on the advantages and disadvantages with time- and eventtriggered control started, e.g., [2], [3], [4], [5], [6], [7].

The goal of this paper is to study a scenario in which multiple event-triggered control loops share a common contention-based communication medium, as illustrated in Fig. 1. The contention-based medium access diminishes the performance of the event-triggered schemes because it leads to collisions. We are motivated by the question: What amount of packet losses can the eventtriggered sampling scheme endure and still perform no worse than a comparable TDMA scheme?

Since event-triggered control involves asynchronously generated sampling times, the overall closed-loop performance depends tightly on the coupling between the loops through the medium. For the class of integrator plants, we derive an explicit expression for how the control criterion depends on the triggering levels and the packet loss probability. For multiple control loops, this result is used to derive a design guideline on how to assign the levels for using the available communication resources optimally. We treat event-triggered loops with contention based MAC as event-triggered loops

This research was partially funded by the Swedish Research Council, the Swedish Foundation for Innovation Systems, and the European Commission.

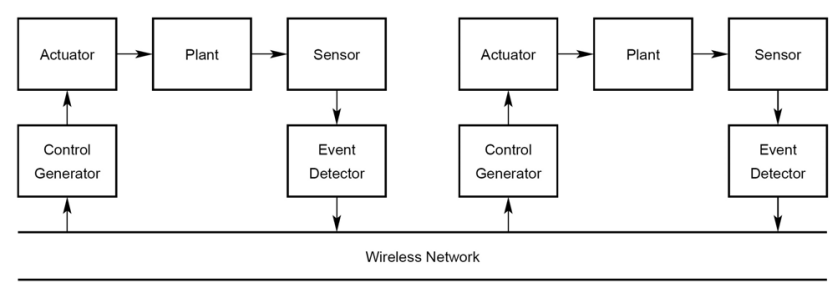

Fig. 1. Event-triggered control of multiple independent loops over a shared contention-based communication medium is considered in the paper.

subject to packet losses. In our model of the MAC, the packet losses happen only because of contention for the medium. But in our analysis, we use an approximation for the statistical properties of packet losses, an approximation which implies that the losses encountered by different loops are independent. We further assume that the sequence of losses for an individual loop is an IID sequence. Under this model for the losses, we study the performance of Delta sampling for the impulse control of Brownian motion.

\section{Problem FORMUlation}

\section{A. Impulse control problem}

Consider a problem of minimum variance impulse control for a noisy integrator. The state signal obeys the drift-free, affine dynamics:

$$
d x_{t}=d B_{t}+u_{t} d t, x(0)=x_{0},
$$

where, $B_{t}$ is a standard Brownian motion process and the control signal $u_{t}$ is a sequence of impulses (DiracDelta functions), and, its time-evolution will be described shortly. The sensor continuously observes the state signal and chooses sampling times when it transmits current values of the state to the actuator. Denote the set of sensor sampling times by the increasing sequence:

$$
\left\{\tau_{0}, \tau_{1}, \tau_{2}, \ldots\right\}
$$

with the requirement that these sampling times are chosen based on causal information about the trajectory of $x_{t}$. This means that, for every positive integer $i$, the sampling time $\tau_{i}$ is a stopping time w.r.t. the filtration $\mathcal{F}_{t}^{x}$. We also require these times to have finite expected values although the sequence of the expected values can and must diverge. In addition, the mean interval between successive sample times should also be 
greater than a strictly positive lower limit. This prevents the possibility of generating with positive probability, an infinite number of samples within a finite interval. Lastly, we arbitrarily set the first sample time $\tau_{0}$ to be zero.

The event-detector at the sensor end determines the sampling times according to chosen rules. At these times, the sensor sends a data packet with the timestamp and also the sample-value of the state. Strictly speaking, the sample value transmitted is the limit from the left of the state trajectory as time approaches the sampling instant $\tau_{i}$ :

$$
x_{\tau_{i}-}=\lim _{\epsilon \rightarrow 0} x_{\tau_{i}-|\epsilon|} .
$$

We need the limit from the left because, at sampling times, the state is potentially reset instantaneously to the origin by the application of an impulse control. The data packet from the sensor containing the time-stamp and the sample value is assumed to have enough bitlength so that the effects of quantization of time-stamps and of sample values can be ignored. We further assume that while some of the sensor's transmitted packets can be lost, whenever delivered, they are delivered with zero delay.

If a data packet from the sensor is received successfully by the actuator, then the sampling time is also the instant when a state-resetting impulse control is applied. Denote the subset of sensor sampling times when the actuator successfully receives the sensor's data packet by the increasing sequence

$$
\left\{\rho_{0}, \rho_{1}, \rho_{2}, \ldots\right\} .
$$

Then, the control signal can be described as follows:

$$
u_{t}=\sum_{n=0}^{\infty}\left(-x_{\rho_{n}}\right) \times \delta\left(\rho_{n}\right) .
$$

Here, $\delta(\cdot)$ is the Dirac delta function. Some control problems actually involve impulse control but a majority involve bounded magnitude control signals. On the other hand, sampling problems for estimation reduce to impulse control for minimizing the variance of the estimation error signal [3].

The control performance is measured through the average power of the state signal:

$$
J=\limsup _{M \rightarrow \infty} \frac{1}{M} \int_{0}^{M} \mathbb{E}\left[x_{s}^{2}\right] d s .
$$

The communication cost is measured through the average sample transmission rate at the sensor:

$$
R_{\tau}=\limsup _{M \rightarrow \infty} \frac{1}{M} \int_{0}^{M} \mathbb{E}\left[\sum_{n=0}^{\infty} \mathbf{1}_{\left\{\tau_{n} \leq M\right\}} \delta\left(s-\tau_{n}\right)\right] d s .
$$

Thus, we do not penalize the strengths of the impulses but only the rate at which they are applied. Roughly speaking, the control cost $J$ decreases as the average sampling rate $R_{\tau}$ is increased. The goal of the designer is to minimize the control cost while ensuring that the communication cost is kept below a desired bound.

Next, we will describe the contention based medium access mechanism and will sketch a model for it.

\section{B. Contention based $M A C$}

We adopt a basic model of sharing the communication medium between multiple, independent communication links. Each transmitter is unsophisticated and cannot sense the presence of carrier signals transmitted by other transmitters. This clearly leads to collisions.

The WirelessHART protocol [8] provides for both TDMA and contention based medium access. The contention based schemes are provided as a means of efficiently using the available bandwidth using eventtriggered communication strategies. Our problem formulation is motivated by this provision in WirelessHART. In such protocols, it is important to determine under what conditions the TDMA scheme is appropriate and under what circumstances the eventtriggered sampling and control schemes provide more efficiency.

To study the performance of multiple event-triggered loops sharing a common medium using contention based access, it is necessary to study the effect of packet losses on event-triggered loops. In the event-triggered control of multiple loops, when the number of such loops sharing a common medium is increased, all other parameters and policies being fixed, so does the frequency of packet collisions in the communication medium. It is also important to know how many separate loops event-triggered strategies can manage in the context of protocols like WirelessHART. This will lead us to study the scalability of control operation for event-triggered schemes. To focus on the effect of shared medium access and the question of scalable operation, we only treat packet losses due to contention. The medium itself is assumed to be reliable when not shared.

We have $N$ control loops each of which is an impulsecontrolled integrator plant. The driving noises at the various plants are mutually independent of each other. The sensors in the loops generate samples for transmission over a shared communications channel to the corresponding actuator nodes, see Figure 1 . The $N$ transmitters contend for the channel. For the sake of simplicity, we consider a slotted system with slot length $L$. After the sample is generated, it is transmitted in the next arriving slot. There is no carrier-sensing and so if more than one sensor generates a sample within a single slot, there will be a collision when the packets are broadcast in the succeeding slot. When a collision happens, the actuators do not take notice. We will approximate the statistics of these collisions by assuming that each loop sees an IID process of packet losses with the average loss rate depending on its sampling strategy as well as that of the other competing loops. We will also consider the loss processes for different loops to be independent of each other although they typically will not be.

The sensors have no retransmissions of packets lost and merely wait for the next sample to be generated as if all previously generated packets have been successfully delivered. Next, we will describe the transmission characteristics of the two sampling methods. 


\section{Periodic AND LEVEL-TRIGGered SAMPling}

We review the main properties of periodic sampling before describing a natural class of event-triggered sampling schemes namely, level-triggered sampling.

\section{A. Periodic sampling}

Under periodic sampling, the sample times for each loop are generated according to a deterministic, periodic sequence:

$$
\tau_{n}=n T+\text { skew }(\text { loop ) for } n \geq 0,
$$

where, $T$ is the common sampling period of all loops and the quantity skew (loop) is an offset which is fixed for a given loop. By assigning these offsets to be different, in fact as an arithmetic progression with common difference no smaller than the slot length $L$, we can avoid collisions. Thus by staggering the transmissions from different loops, periodic sampling can employ TDMA and avoid collisions altogether. It is of course another matter that some packets of the periodic sequence from a sensor may not have much value for the control task and that will be the primary concern of the eventtriggered sampling method.

The periodic sampling scheme can lower its sampling period $T$ up to the product $N L$. Clearly, the limiting factors for increased date rate, equivalently control performance, are the slot length and the number of loops sharing the medium. Since there are no packet losses due to collisions, the state evolution for each loop can be described as follows. We have $\forall n \in \mathbb{Z}^{+}$,

$$
\begin{aligned}
& x_{n T^{+}}=0, \\
& x_{t}=B_{t}-B_{n T}, \forall t \in[n T,(n+1) T) .
\end{aligned}
$$

Here, $B_{t}$ is of course the driving Brownian motion process corresponding to the given loop. The average state distortion and the average sampling rate of the periodic sampling scheme which has no collisions are:

$$
J_{\text {Periodic }}=\frac{T}{2}, \quad R_{\text {Periodic }}=\frac{1}{T} .
$$

\section{B. Level-triggered sampling}

Level-triggered impulse control, which is also called Lebesgue sampling [2], uses the idea that the state should be reset to the origin only when it wanders "sufficiently" far from it. A sample is generated whenever the state trajectory makes a fresh-crossing of a chosen level. The sensor reports the sequence of fresh-crossings to the actuator.

When there are no packet losses, we would only need two symmetric levels: $+\Delta$, and, $-\Delta$. Since some of the sensor's samples could be lost in transmission, the state could wander beyond the levels crossed earlier and potentially "get away" from the origin. Hence, because of the packet losses, we need an infinite set of levels to prevent the escape to infinity from being an event with probability more than zero.

Let the countable set $\mathcal{L}$ denote a chosen infinite, ordered collection of levels which are real numbers:

$$
\left\{\ldots, l_{-2}, l_{-1}, l_{0}, l_{1}, l_{2}, \ldots\right\},
$$

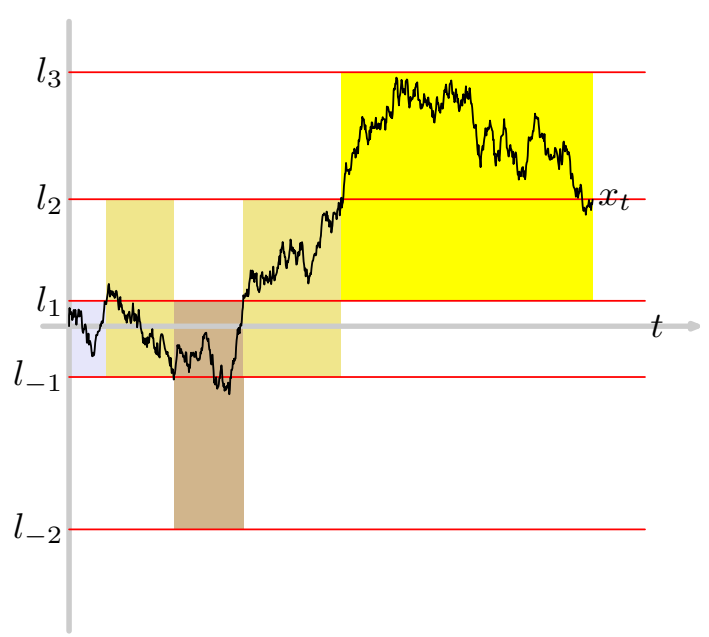

Fig. 2. Level-triggered reset under packet losses. The state is reset to zero only when the the data packet has been successfully received.

where, $\forall i \in \mathbb{Z}, l_{i}<l_{i+1}$. Then the sensor sampling times are given recursively by the fresh-crossings of levels in $\mathcal{L}$ :

$$
\begin{aligned}
\tau_{0} & =0, \\
\tau_{i+1} & =\inf \left\{t \mid t>\tau_{i}, x_{t^{-}} \in \mathcal{L}, x_{t^{-}} \neq x_{\tau_{i}^{+}}\right\}, \forall i \in \mathbb{N} .
\end{aligned}
$$

We have to use fresh crossings instead of arbitrary crossings to keep the sampling rate finite. The insistence on fresh crossings also reflects the key idea of eventtriggered sampling, i.e. send new data packets only if sufficiently new information is available. Notice also that the sequence of inter-sample times $\tau_{i+1}-\tau_{i}$ is a sequence of independent random variables.

\section{Delta sampling}

An important example of level-triggered sampling is Delta sampling where the levels in $\mathcal{L}$ form a lattice with spacing $\Delta$ :

$$
l_{i}=i \Delta, \forall i \in \mathbb{Z}
$$

When there are no sample losses in a loop, Delta sampling is the optimal way to sample scalar Brownian motion in the sense that, among all causal sampling schemes, it minimizes an average cost which is a Lagrangian composed of the the control performance cost $J$ and the communication cost $R_{\tau}$ [3]. Even when there are packet losses, there are important special cases for which we expect delta sampling to be optimal. Such a special case is the problem of average cost estimation [3] with packet losses, but with the restriction that the sequences of losses is not visible to the sensor. This problem arises when the actuator does not acknowledge receipt of packets it receives. Denote the random time required for the state to either increase or decrease by exactly a given positive $\Delta$.

$$
\tau_{\Delta}=\inf \left\{t \mid t \geq 0, x_{t} \notin\left(x_{0}-\Delta, x_{0}+\Delta\right)\right\} .
$$

The inter-sampling times which form an IID sequence have the same distribution as $\tau_{\Delta}$. Hence, it is of interest to obtain its PDF. 


\section{A. The statistics of the inter-sample time}

The PDF of $\tau_{\Delta}$ is given through the solution to the Kolmogorov forward equation. Consider a $C^{2}$ function $f(x, t)$ satisfying the PDE:

$$
\frac{\partial}{\partial t} f(x, t)=\frac{1}{2} \frac{\partial^{2}}{\partial x^{2}} f(x, t),
$$

with the boundary conditions:

$$
\begin{gathered}
f( \pm \Delta, s)=0, \forall s \in[0, \infty), \\
f(x, 0)=\delta(x),
\end{gathered}
$$

where $\delta(x)$ is the Dirac-delta function. The PDF of the stopping time $\tau_{\Delta}$ does not have a closed form solution. There exists a series representation we can find in page 99 of Karatzas and Shreve [9], which is:

$$
f_{\tau}(t)=\Delta \sqrt{\frac{2}{\pi t^{3}}} \sum_{k=-\infty}^{\infty}(4 k+1) e^{-\frac{(4 k+1)^{2} \Delta^{2}}{2 t}} .
$$

This series is not of much use because it is not an integrable series. But we can solve the Kolmogorov backwards equation which is akin to the Cauchy problem of equations $(3,4,5)$, with the appropriate boundary conditions to obtain the average distortion $J_{\Delta}^{\text {No Losses }}$ and the average sensor sampling rate $R_{\Delta}$ :

$$
\begin{aligned}
J_{\Delta}^{\text {No Losses }} & =\frac{\mathbb{E}\left[\int_{0}^{\tau_{\Delta}} x_{s}^{2} d s\right]}{\mathbb{E}\left[\tau_{\Delta}\right]} & =\frac{\Delta^{2}}{6} \\
R_{\Delta} & =\frac{1}{\mathbb{E}\left[\tau_{\Delta}\right]} & =\frac{1}{\Delta^{2}}
\end{aligned}
$$

\section{Delta-triggered impulse Control under IID PACKET LOSSES}

When there are no packet losses, the state is reset to zero whenever the magnitude of the state equals or exceeds $\Delta$. However, when there are losses, the actuator has no knowledge of the sampling event or of the packet loss. The state is reset only when a packet containing a sample is successfully received by the actuator.

Let the increasing sequence of times when packets are successfully received be denoted:

$$
\left\{\rho_{0}=0, \rho_{1}, \rho_{2}, \ldots\right\} .
$$

Clearly it is a thinned version of the sequence of sensor sampling times, and, its average rate of packet receptions is

$$
\begin{aligned}
R_{\rho} & =\limsup _{M \rightarrow \infty} \frac{1}{M} \int_{0}^{M} \mathbb{E}\left[\sum_{n=0}^{\infty} \mathbf{1}_{\left\{\rho_{n} \leq M\right\}} \delta\left(s-\rho_{n}\right)\right] d s, \\
& =p \cdot R_{\tau} .
\end{aligned}
$$

The sequence of intervals between successful receptions of packets is IID. Let $\rho_{p, \Delta}$ be a random variable with the same distribution as this IID sequence. Then the control cost under IID packet losses is given as:

$$
J_{p}=\limsup _{M \rightarrow \infty} \frac{1}{M} \int_{0}^{M} \mathbb{E}\left[x_{s}^{2}\right] d s=\frac{\mathbb{E}\left[\int_{0}^{\rho_{p, \Delta}} x_{s}^{2} d s\right]}{\mathbb{E}\left[\rho_{p, \Delta}\right]} .
$$

We can compute this ratio as a function of the loss rate $p$. We can also infer that the control distortion is bounded for all non-zero rates of packet reception.
Proposition 1 Under IID losses with loss rate $p$, the control cost $J_{p}$ of delta-triggered impulse control for scalar Brownian motion is

$$
J_{p}=\frac{\Delta^{2}(5 p+1)}{6(1-p)}
$$

Proof: We first pay attention to the denominator of equation (7). Without loss of generality, we can consider the situation where $\rho_{1}=\rho_{p, \Delta}$. There, we have,

$$
\begin{aligned}
\mathbb{E}\left[\rho_{p, \Delta}\right] & =\sum_{i=1}^{\infty} \mathbb{E}\left[\tau_{i}\right] \mathbb{P}\left[\rho=\tau_{i}\right], \\
& =\sum_{i=1}^{\infty} i \mathbb{E}\left[\tau_{\Delta}\right] \mathbb{P}\left[\rho=\tau_{i}\right], \\
& =(1-p) \mathbb{E}\left[\tau_{\Delta}\right] \sum_{i=1}^{\infty} i p^{i-1}, \\
& =\frac{\Delta^{2}}{1-p}
\end{aligned}
$$

Now, we tackle the numerator of equation (7).

$$
\begin{aligned}
\mathbb{E}\left[\int_{0}^{\rho_{p, \Delta}} x_{s}^{2} d s\right] & =\sum_{i=1}^{\infty} \mathbb{E}\left[\int_{0}^{\tau_{i}} x_{s}^{2} d s\right] \mathbb{P}\left[\rho=\tau_{i}\right] \\
& =(1-p) \sum_{i=1}^{\infty} p^{i-1} \sum_{n=1}^{i} \mathbb{E}\left[\int_{\tau_{n-1}}^{\tau_{n}} x_{s}^{2} d s\right]
\end{aligned}
$$

To proceed further, we need to compute the term $\nu_{n}=$ $\mathbb{E}\left[\int_{\tau_{n-1}}^{\tau_{n}} x_{s}^{2} d s\right]$ for different possible values of the the run of losses $n$. We have:

$$
\begin{aligned}
\nu_{n} & =\mathbb{E}\left[\int_{\tau_{n-1}}^{\tau_{n}} x_{s}^{2} d s\right] \\
& =\mathbb{E}\left[x_{\tau_{n-1}}^{2} \int_{\tau_{n-1}}^{\tau_{n}} d s+\int_{\tau_{n-1}}^{\tau_{n}}\left(x_{s}-x_{\tau_{n-1}}\right)^{2} d s\right] .
\end{aligned}
$$

Let $\left\{\theta_{i}\right\}$ be an infinite sequence of binary IID variables. Let $\theta$ take vales in $\{-1,+1\}$ with equal probabilities. Then, we can say that the following random variables are equal in probability law:

$$
x_{\tau_{n}} \stackrel{\mathrm{d}}{=} \sum_{m=1}^{n} \theta_{m} \Delta \quad \forall n \in \mathbb{N} .
$$

This enables us to say that

$$
\begin{aligned}
\nu_{n} & =\mathbb{E}\left[\left(\sum_{m=1}^{n} \theta_{m} \Delta\right)^{2}\right] \mathbb{E}\left[\tau_{\Delta}\right]+\mathbb{E}\left[\int_{0}^{\tau_{\Delta}} x_{s}^{2} d s \mid x_{0}=0\right] \\
& =(n-1) \Delta^{4}+\frac{\Delta^{4}}{6}
\end{aligned}
$$


Hence, the numerator of equation (7) becomes

$$
\begin{aligned}
\mathbb{E}\left[\int_{0}^{\rho_{p, \Delta}} x_{s}^{2} d s\right] & =\sum_{i=1}^{\infty}(1-p) p^{i-1} \sum_{n=1}^{i} \frac{\Delta^{4}}{6}+(n-1) \Delta^{4} \\
& =(1-p) \Delta^{4} \sum_{i=1}^{\infty} p^{i-1}\left(\frac{i}{6}+\frac{i(i-1)}{2}\right) \\
& =(1-p) \frac{\Delta^{4}}{6} \sum_{i=1}^{\infty} p^{i-1}\left(3 i^{2}-2 i\right) \\
& =\frac{\Delta^{4}(5 p+1)}{6(1-p)^{2}}
\end{aligned}
$$

Combining this last equation with (9) we obtain,

$$
J_{p}=\frac{\nu_{n}}{\mathbb{E}\left[\rho_{p, \Delta}\right]}=\frac{\Delta^{2}(5 p+1)}{6(1-p)} .
$$

This completes the proof.

Let us note how this average distortion compares with that of periodic sampling under IID losses and identical communication costs. Sampling with a fixed period $T$ and with the loss rate of $p$ incurs an average distortion of

$$
\frac{T(1+p)}{2(1-p)}
$$

For a fair comparison, we should force the base sampling rates of the periodic and Lebesgue schemes to be the same:

$$
T=\Delta^{2}
$$

Notice then that for every $p<1$, the distortion of the deterministic sampler is always higher than the distortion due to the equally spaced Lebesgue sampler.

On the other hand, periodic sampling schemes are well-suited for TDMA based medium access and so can avoid or at least drastically reduce packet losses. The interesting question then is: At what packet loss rate for the Lebesgue scheme does the TDMA scheme with no losses start becoming better ? The answer is easy to compute:

$$
\begin{aligned}
& \frac{(1+5 p)}{3(1-p)} \geq 1, \\
\Leftrightarrow & p \geq 0.25 .
\end{aligned}
$$

\section{Multiple loops Sharing the same medium}

When $N$ independent loops contend and create collisions, the processes of packet losses across the different loops are not independent. The independence is lost through the coupling caused by competing for the same channel. If the samples from two or more sensors collide within a single packet slot, then the future sample times of these particular sensors are correlated.

However, we will adopt an approximation which ignores this dependence. Inspired by the properties of sampling according to independent Poisson processes we assume that the loss processes across the different loops are independent. For Poisson streams, for a particular sensor, the sample streams of the other sensors only matter through their average behaviour.
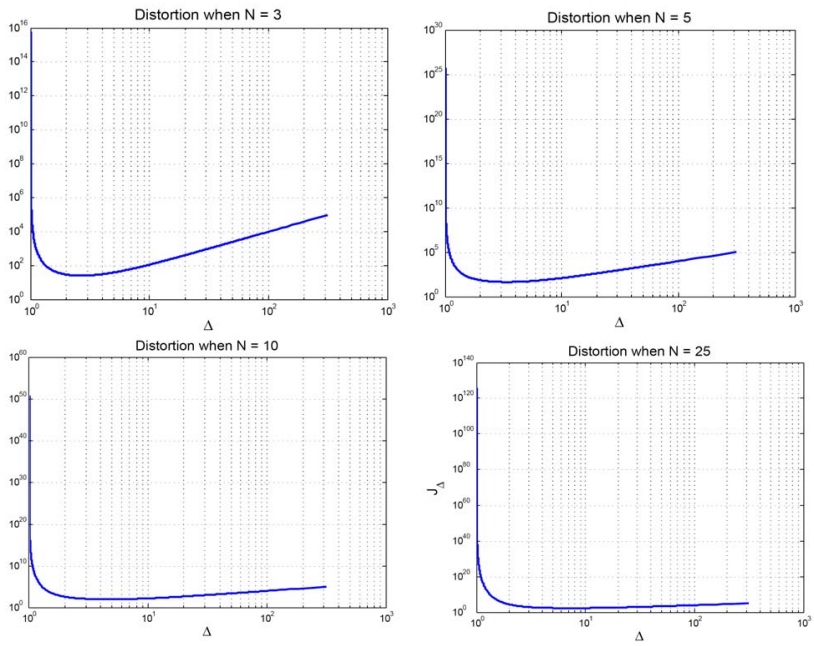

Fig. 3. Trade off between high collisions and slow sampling with $N$ competing loops.

This property is called PASTA (Poisson arrivals see time averages [11]).

Under this assumption of independent losses across the different loops, the loss probability can be described as the likelihood that a sample generated in one loop faces at least one competing transmission from another loop:

$$
p=1-\left(1-\frac{L}{\Delta^{2}}\right)^{N-1}
$$

To decrease the loss rate, one should increase $\Delta$. On the other hand, increasing $\Delta$ leads to decreasing the sampling rate at the sensor and since less samples are generated to begin with, there is the risk that too few samples get across. This trade-off [10] is nicely captured in the expression for distortion obtained by combining equations (8) and (11):

$$
\tilde{J}_{\Delta}=\frac{L\left(6-5 \phi^{N-1}\right)}{6 \phi^{N-1}(1-\phi)},
$$

where,

$$
\phi=1-\frac{L}{\Delta^{2}} .
$$

Figure (3) shows plots of the control performance with $3,5,10$ or 25 competing control loops. The $\mathrm{x}$-axis in these plots is the normalized threshold: $\frac{\Delta}{\sqrt{L}}$. Notice the presence of the minimum representing the best tradeoff between minimizing collisions and maximizing the sampling rate at the sensor. The choice of threshold achieving the minimum increases monotonically with the number of competing loops. The minimizing value of $\Delta$ satisfies a polynomial equation of degree $N-1$ and does not have a closed form solution. However the plots in figure (3) can be used to find it.

\section{A. Scalability w.r.t. $N$}

When there are no packet losses, for the same sampling rate, Delta sampling incurs only a third of the cost of the periodic scheme. However when there is competition for access to the medium, the packet loss 


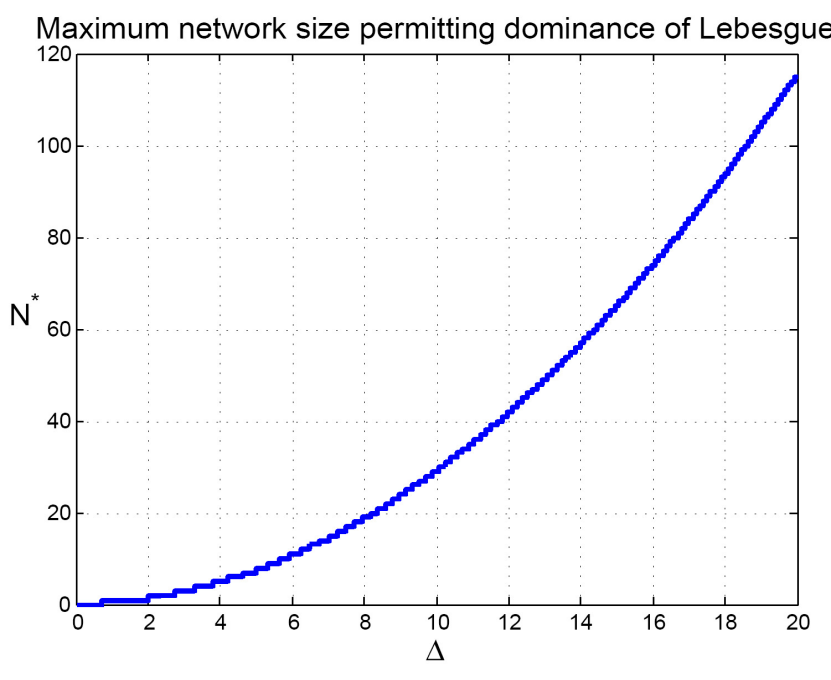

Fig. 4. The maximum number of loops for which the equally spaced Lebesgue scheme dominates periodic control.

rate is non-zero and the efficiency gain of Lebesgue schemes erodes away. Here, we determine the number of loops $N$ for which Delta sampling can handle the losses due to contention and still perform no worse than the TDMA scheme.

The critical loss rate grows monotonically as a function of the number of competing loops $N$. Let $N^{*}$ be the limit beyond which the loss probability is such that Lebesgue sampling scheme loses its efficiency advantage over the TDMA-based resetting scheme. We can write

$$
N^{*}=1+\left\lfloor\frac{\log (0.75)}{\log \left(1-\frac{L}{\Delta^{2}}\right)}\right\rfloor .
$$

The monotonic growth of $N^{*}$ as a function of the threshold $\Delta$ is depicted in figure (4). It also makes it clear that if the required sampling rates for the control loops are rather low, then the the Lebesgue scheme can tolerate a lot of crowding while still maintaining better performance than the TDMA-based one.

\section{Conclusions and Open Problems}

We have considered event-triggered impulse control under lossy communication. In specific, we studied impulse control triggered by delta sampling with IID losses of the samples. It is shown for scalar integrator plants how the average state variance depends on $\Delta$ and the packet loss rate. For multiple control loops, this result is used to derive a design guideline on how to assign the levels and the available communication resources. It is of high interest to pursue this line of research and investigate event-triggered schemes with added mechanisms for coping with packet losses. One possibility is to sample according to a hybrid scheme with both event-triggered and occasional periodically samples. The periodic sample times act as deterministic time-outs that can be scheduled to avoid collisions.

It is also interesting to study event-triggered sampling schemes for control loops where ACK packets are available. Then the sensor can detect packet losses and modify its sampling strategies suitably. Consider the sampling scheme based on Delta up-crossings:

$$
\begin{aligned}
\tau_{0}^{\Uparrow} & =0, \\
\tau_{i+1}^{\Uparrow} & =\inf \left\{t: t>\tau_{i},\left|x_{t^{-}}\right|-\left|x_{\left(\tau_{i}^{\Uparrow}\right)^{+}}\right| \geq \Delta,\right\}, \forall i \in \mathbb{N} .
\end{aligned}
$$

For a packet loss rate of $p$, the mean sensor sampling rate (rate of up-crosssings in the magnitude of $x_{t}$ ) $R^{\Uparrow}$, and, the average control cost $J^{\Uparrow}$ can be computed to be:

$$
\begin{aligned}
R^{\Uparrow} & =\frac{1-p}{p \Delta^{2}} \ln \frac{1}{1-p}, \\
J^{\Uparrow} & =\frac{(1-p) \Delta^{2}}{6(1+p)}\left(\frac{24}{(1-p)^{3}}-\frac{36}{(1-p)^{2}}+\frac{14}{1-p}-1\right) .
\end{aligned}
$$

How can we use formulas like these ? Suppose that the packet loss rate is given. If the communication cost is prescribed, then the minimum $\Delta$ to be used is automatically fixed.

On the other hand, suppose that the packet rate is not fixed but we are free to vary it by varying the number of loops $N$. How do we choose $\Delta$ and $p$ for the up-crossings scheme to obtain the best possible ratedistortion curve ? Furthermore, consider an alternative sampling scheme where the threshold $\Delta$ can be varied for different samples. In this scheme, when the sensor is notified of a loss, how should it choose

$$
\Delta=\Delta \text { (number of samples since last } \mathrm{ACK})
$$

used to generate the next sample?

\section{REFERENCES}

[1] H. Kopetz, Real-Time Systems: Design Principles for Distributed Embedded Applications. Norwell, MA, USA: Kluwer Academic Publishers, 1997.

[2] K. J. Åström and B. Bernhardsson, "Comparison of Riemann and Lebesgue sampling for first order stochastic systems," in Proceedings of the 41st IEEE conference on Decision and Control (Las Vegas NV, 2002), 2002, pp. 2011-2016.

[3] M. Rabi, "Packet based inference and control," Ph.D. dissertation, University of Maryland, College Park, Sep 2006.

[4] M. Rabi, G. V. Moustakides, and J. S. Baras, "Multiple sampling for estimation on a finite horizon," in Proceedings of the 45th IEEE conference on Decision and Control (San Diego, CA, 2006), 2006, pp. 1351-1357.

[5] E. Johannesson, T. Henningsson, and A. Cervin, "Sporadic control of first-order linear stochastic systems," in Proc. 10th International Conference on Hybrid Systems: Computation and Control, ser. Lecture Notes in Computer Science 4416. Pisa, Italy: Springer-Verlag, Apr. 2007.

[6] M. Rabi, K. H. Johansson, and M. Johansson, "Optimal stopping for event-triggered sensing and actuation," in Proceedings of the 47th IEEE Conference on Decision and Control (Cancun, Mexico, 2008), December 2008.

[7] A. Cervin and T. Henningsson, "Scheduling of event-triggered controllers on a shared network," in Proc. 47th IEEE Conference on Decision and Control, Cancun, Mexico, dec 2008, to appear.

[8] WirelessHART consortium, "WirelessHART protocol specification," HART communication foundation, Tech. Rep., April 2008. Available: http://www.hartcomm2.org/hart protocol/wireless hart/wireless hart main.html

[9] I. Karatzas and S. E. Shreve, Brownian motion and stochastic calculus, 2nd ed., ser. Graduate Texts in Mathematics. New York: Springer-Verlag, 1991, vol. 113.

[10] M. Rabi, L. Stabellini, A. Proutiere and M. Johansson, Networked estimation under contention-based medium access, to appear in the International journal on robust and nonlinear control.

[11] R. W. Wolff, "Poisson arrivals see time averages," Operations Research, vol. 30, no. 2, pp. 223-231, 1982. 